\title{
Characterization of an Electromagnetic Calorimeter for the Proposed International Linear Collider
}

\author{
Merideth Frey \\ Office of Science, SULI Program \\ Wellesley College \\ Stanford Linear Accelerator Center \\ Menlo Park, California
}

August, 242006

Prepared in partial fulfillment of the requirements of the Office of Science, U.S. Department of Energy’s Science Undergraduate Laboratory Internship under the direction of Dr. Norman Graf and Dr. Tony Johnson in the International Linear Collider group of the High Energy Physics division at Stanford Linear Accelerator Center.

Participant:

Signature

Research Advisor:

Signature 


\section{Table of Contents}

Abstract 3

$\begin{array}{ll}\text { Introduction } & 4\end{array}$

Materials and Methods $\quad 5$

$\begin{array}{ll}\text { Results } & 7\end{array}$

$\begin{array}{lr}\text { Discussion and Conclusion } & 9\end{array}$

$\begin{array}{ll}\text { Acknowledgements } & 12\end{array}$

$\begin{array}{ll}\text { References } & 12\end{array}$

$\begin{array}{ll}\text { Tables } & 14\end{array}$

$\begin{array}{ll}\text { Figures } & 15\end{array}$ 


\begin{abstract}
Characterization of an Electromagnetic Calorimeter for the Proposed International Linear Collider. MERIDETH FREY (Wellesley College, Wellesley, MA 02481) NORMAN GRAF (Stanford Linear Accelerator Center, Menlo Park , CA 94025) TONY JOHNSON (Stanford Linear Accelerator Center, Menlo Park , CA 94025).
\end{abstract}

The International Linear Collider (ILC) is part of a new generation of accelerators enabling physicists to gain a deeper understanding of the fundamental components of the universe. The proposed ILC will accelerate positrons and electrons towards each other with two facing linear colliders, each twenty kilometers long. Designing and planning for the future accelerator has been undertaken as a global collaboration, with groups working on several possible detectors to be used at the ILC. The following research at the Stanford Linear Accelerator Center (SLAC) pertained to the design of an electromagnetic calorimeter. The energy and spatial resolution of the calorimeter was tested by using computer simulations for proposed detectors. In order to optimize this accuracy, different designs of the electromagnetic calorimeter were investigated along with various methods to analyze the data from the simulated detector. A low-cost calorimeter design was found to provide energy resolution comparable to more expensive designs, and new clustering algorithms offered better spatial resolution. Energy distribution and shape characteristics of electromagnetic showers were also identified to differentiate various showers in the calorimeter. With further research, a well-designed detector will enable the ILC to observe new realms of physics. 


\section{INTRODUCTION}

Particle detectors have changed dramatically from early cloud chambers into the elaborate multi-layered devices used today. With new higher energy colliders revealing particles never seen before, detectors need to be as accurate as possible in order to determine properties of these new particles. The ILC provides an opportunity for physicists to design and build precise detectors that are not useful for present accelerators such as the Large Hadron Collider (LHC). Since electrons and positrons are fundamental particles - unlike the hadrons that will be collided at the LHC - the ILC will involve less complicated particle transformations, making the use of more precise instruments practical [1]. With higher energy collisions and well-designed detectors, the ILC will provide results to further illuminate physics at the fundamental particle level.

Scientists around the world are working together to design the ILC. At the Stanford Linear Accelerator Center (SLAC), the Linear Collider group is designing an electromagnetic (EM) calorimeter - the component of the ILC particle detector optimized to measure the energy and position of electrons and gamma ray photons. The proposed EM calorimeter is heterogeneous and made of multiple layers of materials: a dense metal with which particles interact and lose energy to forming new particles, and an active material which produces a detectable signal dependent upon the amount of energy absorbed. This type of calorimeter only directly measures a small percentage of the particle energy, but since active material is located evenly throughout the calorimeter, researchers can statistically find the overall energy absorbed. Using a heterogeneous calorimeter enables researchers to analyze the signal using various statistical methods and to find an optimal dense metal absorber for the particular calorimeter. In 
this study, heterogeneous calorimeters with different numbers of layers and various thicknesses of dense metal absorbers were analyzed to optimize energy resolution.

Along with the many possible physical components that can be optimized to increase accuracy for a given calorimeter, methods to analyze the data recorded by the detector can also be investigated. When a particle collides with the calorimeter, it produces many other particles which also collide with the calorimeter material, creating what is called a particle shower. When a collision occurs in the active material (referred to as a hit), the position and energy is recorded. This energy is then corrected using statistical analysis to include the energy lost in the passive material. The energy of the original particle is measured by grouping all the hits from the showered particles (referred to as a cluster) and summing their energy. However, deciding what hits belong to what particle is complicated; especially if two showering particles hit the calorimeter close to each other at around the same time. For this reason, different clustering algorithms have been written to try to improve the resolution of two nearby particles and their effectiveness needs to be tested.

Other valuable analyses involve differentiating between electromagnetic showers showers resulting from an electron or photon colliding with the calorimeter - and showers resulting from other particles that interact with the calorimeter. Electromagnetic showers have many identifying features in their shape and energy distribution. Determining these characteristics can help identify relevant electromagnetic showers from the calorimeter data.

\section{MATERIALS AND METHODS}

The software used to simulate the calorimeter for various events was Simulator for the Linear Collider (SLIC) [2] written by Jeremy McCormick who works in the Linear Collider group at SLAC and based the program on the Geant4 simulation toolkit [3]. This software produces data of detected hits from multiple collisions for a given detector which can be 
analyzed using the Java Analysis Studio (JAS). JAS is an open-source, data analysis tool which provides a graphical user interface to load and compile user Java programs. For plotting data, JAS implements Abstract Interfaces for Data Analysis (AIDA). There is also a built-in event display called WIRED4 - see Figure 1 - which allows the user to view the hits and tracks of particles resulting from a collision at any desired angle and display information about any selected item. The Linear Collider Simulator (LCSim) Event Browser displays all the information known about different hits and tracks of particles from a given simulated collision and was mostly used to double-check the plots.

In order to determine the accuracy of the EM calorimeter at measuring the energy of a particle, single particles of known energy and position were used and the energy resolution of three different calorimeter designs was analyzed. One design had thirty layers of the same absorbent material thickness (referred to as homogeneous thirty layer calorimeter), another had forty layers of the same absorbent material thickness (referred to as homogeneous forty layer calorimeter), and the final design had twenty layers of one absorbent material thickness and then ten layers with the absorbent material twice as thick (referred to as heterogeneous thirty layer calorimeter). The absorbent material used for these designs had $95 \% \mathrm{~W}, 4.4 \% \mathrm{Ni}$, and $0.6 \% \mathrm{Fe}$. See Table 1 for the absorbent material thickness for each of the calorimeter designs. Figure 2 shows a diagram of one layer in the calorimeter. Single photons were also used to identify characteristics of electromagnetic showers by examining the photons' shower shapes and energy distributions.

To determine how well the calorimeter could resolve two nearby particles, analyses were done using two equal-energy photons with various opening angles. Spatial resolution is highly dependent on the type of clustering algorithm used to determine what hits came from the same particle, so various clustering algorithms were analyzed. The simplest clustering algorithm - 
called the nearest neighbor - groups all nearby hits into the same cluster. The directed tree algorithm starts clustering at the first layer of the calorimeter and proceeds to higher layers. This method groups hits into the same clusters as the nearby hits in the previous layers. This means that two clusters which overlap in the calorimeter - and would be declared one cluster by the nearest neighbor algorithm - can be differentiated if they both are distinct clusters in the first layer. The fixed cone algorithm finds the highest energy hits in the calorimeter and forms cones around these hits with vertex at the collision point - see Figure 3. It then groups all the hits within a cone as part of one cluster.

All analyses were done using programs written in Java and plotted using AIDA through JAS. All measurements were found by analyzing many events ( 5000) and fitting histograms with a Gaussian or taking the mean. The heterogeneous thirty layer calorimeter was used unless otherwise specified.

\section{RESULTS}

Figures 4, 5, and 6 are plots of the total energy found in the EM calorimeter for a single particle hitting the barrel perpendicularly versus the known energy of the particle using the homogeneous thirty layer calorimeter, homogeneous forty layer calorimeter, and heterogeneous thirty layer calorimeter, respectively. Table 2 gives the values of the constants found from a linear fit of the data for each of the calorimeter designs.

Figure 7 shows a plot of the total cluster energy found for a single $20 \mathrm{GeV}$ photon when varying the input parameters for the nearest neighbor clusterer. The input parameters include the minimum number of hits to form a cluster (minCell) and three parameters that determine how nearby a hit must be to be included in the cluster (dlayer, dU, and dV). The parameter dlayer defines the maximum number of layers away from the cluster a hit can be to be included in the cluster. The parameters $\mathrm{dU}$ and $\mathrm{dV}$ define the maximum distance from the cluster a hit can be in 
either direction within the layer to be included in the cluster. The parameter minCell was kept constant at five and dlayer was kept constant at one. Since photon clusters are known to have circular cross-sections, $\mathrm{dU}$ and $\mathrm{dV}$ were kept equal, but the magnitude for $\mathrm{dU}=\mathrm{dV}$ was varied.

Figures 8, 9, and 10 are energy resolution plots for single photons using the homogeneous thirty layer calorimeter, homogeneous forty layer calorimeter, and heterogeneous thirty layer calorimeter, respectively. For sampling calorimeters, the intrinsic energy resolution $(\sigma / E)$ can be approximated by:

$$
\sigma / \mathrm{E} \approx \mathrm{p} 1 / \mathrm{sqrt}(\mathrm{E})+\mathrm{p} 0
$$

where $\mathrm{p} 1$ and $\mathrm{p} 0$ are constants, $\mathrm{E}$ is the mean of the energy distribution, and $\sigma$ is the standard deviation (see [4] for a more thorough analysis of energy resolution for sampling calorimeters). By plotting $y=\sigma / E$ and $x=s q r t(E)$ and fitting a line to the data, the constants $p 1$ and $p 0$ were found. The nearest neighbor algorithm was used for clustering, with minCell $=5$ and $d U=d V=$ dlayer $=1$. In order to analyze only the cluster formed by the incident photon, the maximumenergy cluster of each event was used to find $\mathrm{E}$ and $\sigma$. Table 3 gives the values of $\mathrm{p} 1$ and $\mathrm{p} 0$ found from a linear fit of the data for each of the calorimeter designs.

Figures 11 and 12 show the total energy and approximated width for a photon cluster in each layer of the electromagnetic calorimeter. Single particle events were used with $20 \mathrm{GeV}$ photons. The clusters were found using the nearest neighbor algorithm, with minCell $=5$ and $\mathrm{dU}$ $=\mathrm{dV}=$ dlayer $=1$. Only clusters with high energy were analyzed so that the many low-energy clusters (usually isolated hits) would not ruin the analysis. This was accomplished by comparing the cluster energy to a threshold energy, usually about $15 \%$ of the known energy of the incident photon. For each high-energy cluster, the total energy in each layer was found and plotted. The width in each layer was approximated by finding the maximum distance from a hit in the cluster 
to the centroid of the cluster along the z-direction (parallel to the axis of the cylindrical detector) and multiplying this value by two.

Figures 13, 14, and 15 show the shape parameters (similar to moments of inertia about three perpendicular axes using energy instead of mass) for a single photon cluster versus energy

of the photon. Different input parameters of the nearest neighbor clustering algorithm were used to see how this affected the shape of the cluster. The parameter minCell was kept constant at five, while $\mathrm{dU}=\mathrm{dV}=$ dlayer was varied.

Figure 16 shows the mean number of clusters detected for two $1 \mathrm{GeV}$ photons with various opening angles using the clustering algorithms: nearest neighbor, directed tree, and fixed cone. Only high-energy clusters were analyzed, and low-energy clusters from isolated hits were filtered out by use of a threshold energy ( $50 \%$ of known photon energy).

\section{DISCUSSION AND CONCLUSION}

The energy resolution properties of the simulated detector can be seen in Figures 4 through 10. The plots of the total energy in the EM calorimeter versus the known energy of the photon for single photon events (Figures 4, 5, and 6) all have linear fits with slopes near to one and small y-offsets (Table 2). As expected, the most expensive design, the homogeneous forty layer calorimeter, performed the best, having a slope of $\sim 0.997$ and an offset of $\sim-0.00336$. The least expensive design, the homogeneous thirty layer calorimeter, performed the worst with a slope of $\sim 0.979$ and an offset of $\sim 0.0170$. The heterogeneous thirty layer calorimeter (a cheaper alternative to the forty layer design) has a slope of $\sim 0.982$ and an offset $\sim 0.0146$, making this design better than the homogeneous thirty layer calorimeter but slightly worse than the more expensive homogeneous forty layer calorimeter. Note that these plots use all the energy detected in the EM calorimeter, and these results are less ideal when clustering is used. 
Figure 7 shows how changing the input variables of the nearest neighbor clustering algorithm can alter energy resolution. Increasing $\mathrm{dU}$ and $\mathrm{dV}$ increases the total cluster energy because more hits are being included. However, this energy appears to plateau for higher values of $d U$ and $d V$, suggesting that eventually increasing $d U$ and $d V$ may cease to be useful for increasing the total cluster energy. For more many particle events, this increase of neighborhood decreases the spatial resolution of showers in the detector because nearby showers may be grouped into one cluster. Further study to find the ideal values for $\mathrm{dU}$ and $\mathrm{dV}$ to increase energy resolution without losing spatial resolution would be beneficial.

Further energy resolution analyses for photons of different energies are shown in Figures 8, 9, and 10. Ideally, the intrinsic energy resolution should be smaller than $20 \%$ of the energy of the particle. For the plots in the figures, the linear fit would have no y-offset and a very low slope. All the calorimeter designs had very similar results (Table 3). Surprisingly, the homogeneous thirty layer calorimeter has the lowest slope and y-offset but the highest $\chi^{2}$ value and the homogeneous forty layer calorimeter has the highest slope and y-offset but the lowest $\chi^{2}$ value. The heterogeneous thirty layer calorimeter has a low slope within the error bounds of the homogeneous thirty layer calorimeter and a lower $\chi^{2}$ value. With these results along with the linearity results, the heterogeneous calorimeter appears to provide a good blend of the thirty and forty layer homogeneous calorimeters without a huge cost increase from the cheaper homogeneous thirty layer calorimeter.

Characteristics of electromagnetic showers, using the nearest neighbor clustering algorithm, are shown in Figures 11 through 15. As can be seen in Figures 11 and 12, electromagnetic showers on average have a very specific energy distribution and width throughout the layers of the calorimeter. This information can be used to differentiate electromagnetic showers from other showers that may occur in the detector. The heterogeneous 
thirty layer calorimeter was used, and the higher absorbency - due to the thicker absorbent material in the last ten layers of the calorimeter - causes the energy measurements to increase dramatically for layers twenty-one through thirty in Figure 11.

The shape parameters plotted in Figures 13, 14, and 15 also show a distinct shape for electromagnetic showers. Shape parameter one is much smaller than shape parameters two and three, which are very similar in magnitude. These shape parameters suggest a shower shape similar to an elongated ellipsoid with a circular cross-section. Of course, the magnitudes of the shape parameters change depending on energy and the input parameters used for the nearest neighbor clustering algorithm. When the energy is increased, the shape parameters are also increased because the shower is larger in all directions. The shower also is larger in all directions when increasing dlayer $=\mathrm{dU}=\mathrm{dV}$ because more hits originally outside the cluster are being included. The dependence of the shape parameters on energy and clustering inputs make these characteristics more difficult to use in identifying electromagnetic showers. However, the ratios between the different shape parameters remain fairly constant and might prove useful for identification. For example, the ratio of shape parameter two to three is still very close to one despite its slight dependence on energy and clustering algorithm inputs.

As can be seen in Figure 16, all the clustering algorithms have a constant mean number of clusters detected for opening angles greater than one degree. Since the means are all near two, all the clustering algorithms were able to resolve the two photons with these opening angles. Despite this non-dependence on opening angle, the nearest neighbor algorithm has a significantly lower mean than the other two more advanced clustering algorithms for opening angles greater than one degree. This suggests that the directed tree and fixed cone clustering algorithms are more successful at resolving two nearby photons on average for these angles. However, for an opening angle of one degree, the mean number of clusters for the nearest neighbor clustering 
algorithm is very similar to the mean number for the larger opening angles, whereas the mean number of clusters for the directed tree and fixed cone clustering algorithms decreased dramatically to a value closer to one. This suggests that for an opening angle of one degree, the direct tree and fixed cone algorithms cannot resolve the two photons.

With this project, many characteristics of a simulated EM calorimeter for the ILC were analyzed. The energy resolutions of three different calorimeter designs were compared and showed that a heterogeneous calorimeter may be a low-cost way to attain good resolution. Characteristics of EM showers were identified that can help differentiate showers in the calorimeter. The spatial resolution using three clustering algorithms was investigated, and the more advanced algorithms, directed tree and fixed cone, out-performed the nearest neighbor algorithm for opening angles greater than one degree. There is still much more research to be done, including using single particle events that hit the detector at various angles and identifying EM showers using different clustering algorithms. With enough analysis, the best possible calorimeter for the ILC can be designed to help detect new and exciting physics.

\section{ACKNOWLEDGMENTS}

This research was conducted at the Stanford Linear Accelerator Center through the Science Undergraduate Laboratory Internship program organized by the U.S. Department of Energy, Office of Science. They have my heartfelt gratitude for providing me with a wonderful learning experience. I thank Ronald Cassell for his help in many areas of my research and Jeremy McCormick for the use of his detector simulation program. Special thanks go to my mentors Norman Graf and Tony Johnson who directed my analyses with enthusiasm and patience and made this a thoroughly enjoyable experience.

\section{REFERENCES}

[1] M. Perricone, "Super-fast Super-sensitive Detectors," in Symmetry - Dimensions of Particle Physics, Vol.2, Is. 07, Sept. 2005, pp. 18-23. 
[2] SLIC documentation and tutorials can be found at: https://confluence.slac.stanford.edu/display/ilc/org.lcsim

[3] J. Allison, et al., "Geant4 Developments and Applications," in IEEE Transactions on Nuclear Science, Vol. 53, No. 1, Feb. 2006, pp. 270-278.

[4] C.W. Fabjan and T. Ludlam, "Calorimetry in High-Energy Physics," in Annual Review of Nuclear and Particle Science, Vol. 32, Dec. 1982, pp. 335-389. 


\begin{tabular}{|l|c|c|}
\hline & \multicolumn{2}{|c|}{ Absorber Thickness (cm) } \\
\hline Homogeneous 30 Layer & \multicolumn{2}{|c|}{0.271} \\
\hline Homogeneous 40 Layer & \multicolumn{2}{|c|}{0.271} \\
\hline Heterogeneous 30 Layer & 0.271 (for layers 1-20) & 0.543 (for layers 21-30) \\
\hline
\end{tabular}

Table 1. Absorber thicknesses in each layer for the different calorimeter designs.

\begin{tabular}{|l|c|c|c|}
\hline & p0 & p1 & $\boldsymbol{\chi}^{\mathbf{2}}$ \\
\hline Homogeneous 30 Layer & 0.017002 & 0.97942 & 43.086 \\
\hline Homogeneous 40 Layer & -0.0033657 & 0.99702 & 2.6931 \\
\hline Heterogeneous 30 Layer & 0.014626 & 0.98242 & 29.274 \\
\hline
\end{tabular}

Table 2. Values of the linear fits for total energy detected in the calorimeter versus known photon energy using different calorimeter designs. The fitted function has the form $\mathrm{p} 0+\mathrm{p} 1 \mathrm{x}$.

\begin{tabular}{|l|c|c|c|}
\hline & p0 & p1 & $\boldsymbol{\chi}^{\mathbf{2}}$ \\
\hline Homogeneous 30 Layer & 0.00014060 & 0.19490 & 16.792 \\
\hline Homogeneous 40 Layer & -0.0021909 & 0.19905 & 6.2012 \\
\hline Heterogeneous 30 Layer & 0.0010234 & 0.19593 & 6.4259 \\
\hline
\end{tabular}

Table 2. Values of the energy resolution linear fits using different calorimeter designs. The fitted function has the form $\mathrm{p} 0+\mathrm{p} 1 \mathrm{x}$. 


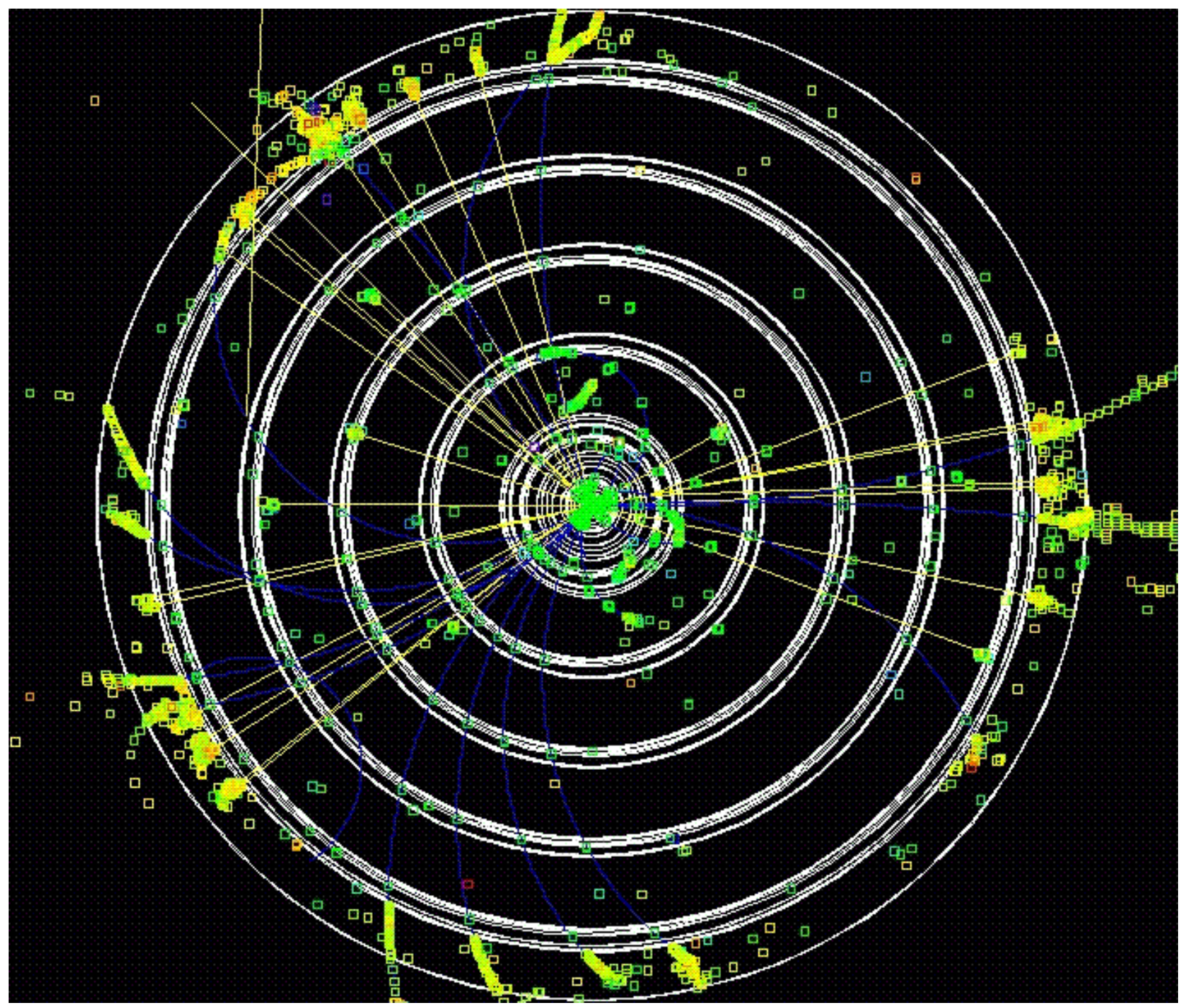

Figure 1. Example of the WIRED4 event display showing a simulated electron-positron collision with tracks of particles and hits detected in the calorimeter.

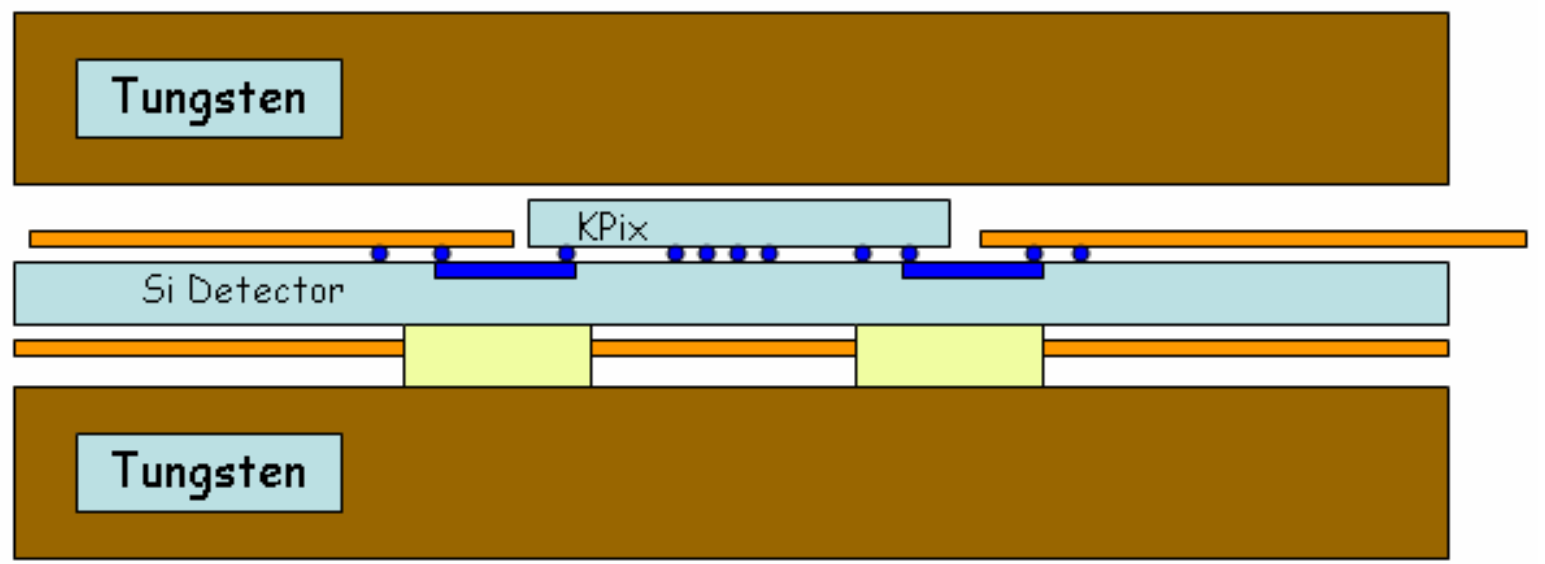

Figure 2. Diagram of a layer in the heterogeneous calorimeters being analyzed in this research. KPix is the readout chip for the detector. 


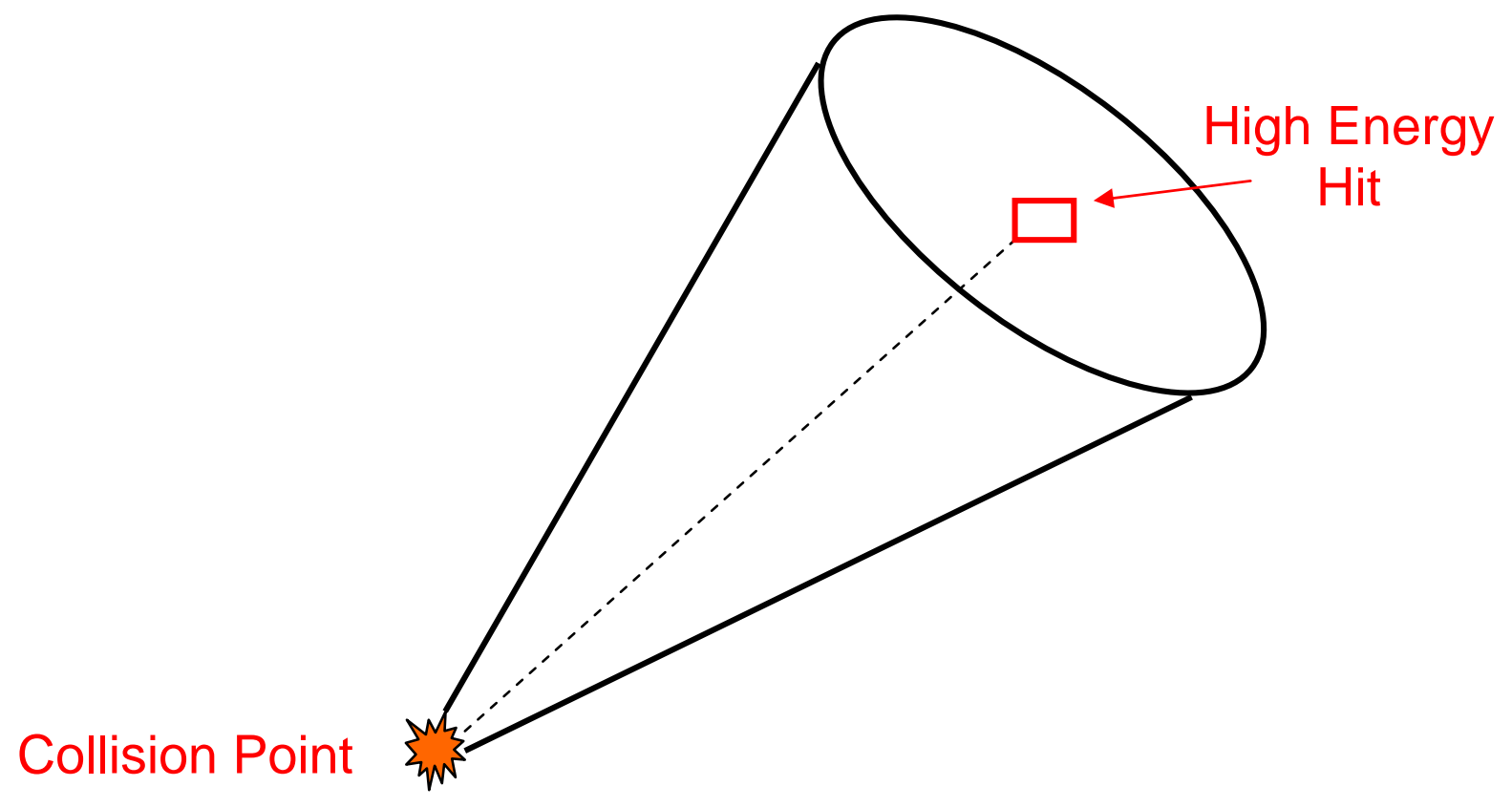

Figure 3. Diagram of the fixed cone clustering algorithm. The algorithm starts looking at the highest energy hits, then draws a cone about this hit with vertex at the collision point. All hits within the cone are grouped together into one cluster. 
Total Energy in the EM Calorimeter vs. Photon Energy with Homogeneous 30 Layer Calorimeter

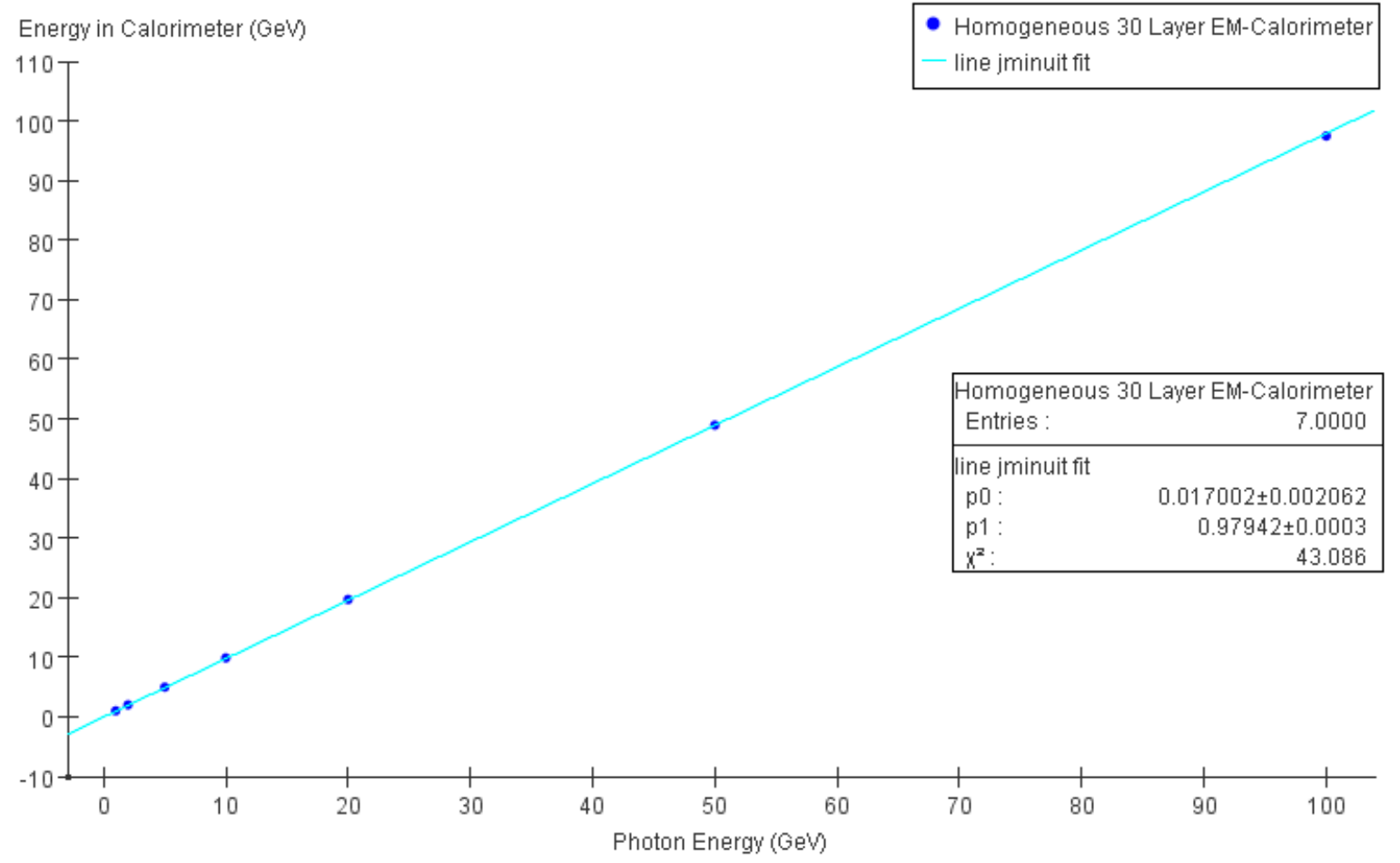

Figure 4. Linearity plot of the total energy in the homogeneous thirty layer EM calorimeter versus the actual energy of a single photon event. The data points are fitted with a linear function of the form $\mathrm{p} 0+\mathrm{p} 1 \mathrm{x}$.

Total Energy in the EM Calorimeter vs. Photon Energy with Homogeneous 40 Layer Calorimeter

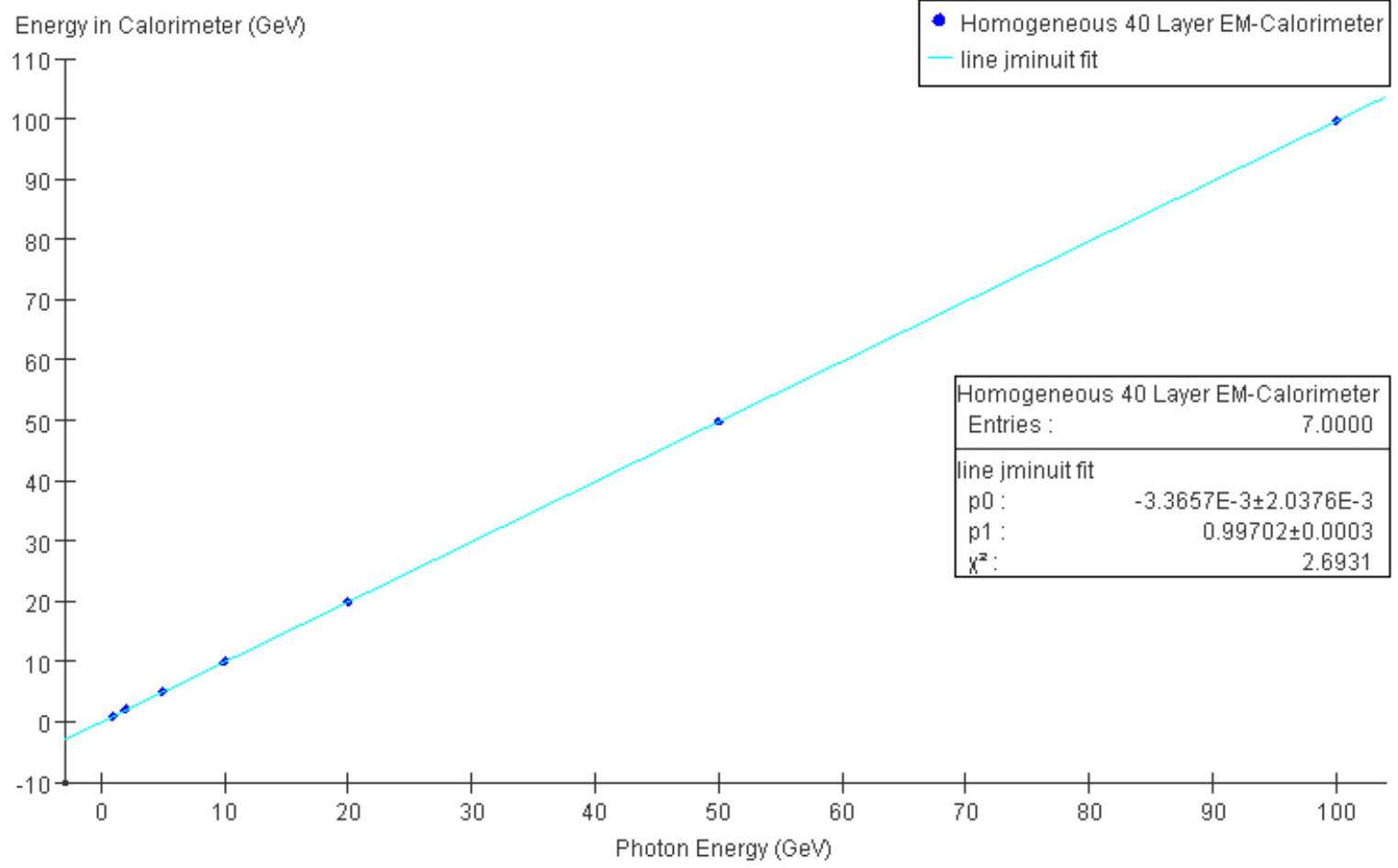

Figure 5. Linearity plot of the total energy in the homogeneous forty layer EM calorimeter versus the actual energy of a single photon event. The data points are fitted with a linear function of the form $\mathrm{p} 0+\mathrm{p} 1 \mathrm{x}$. 
Total Energy in the EM Calorimeter vs. Photon Energy with Heterogeneous 30 Layer Calorimeter

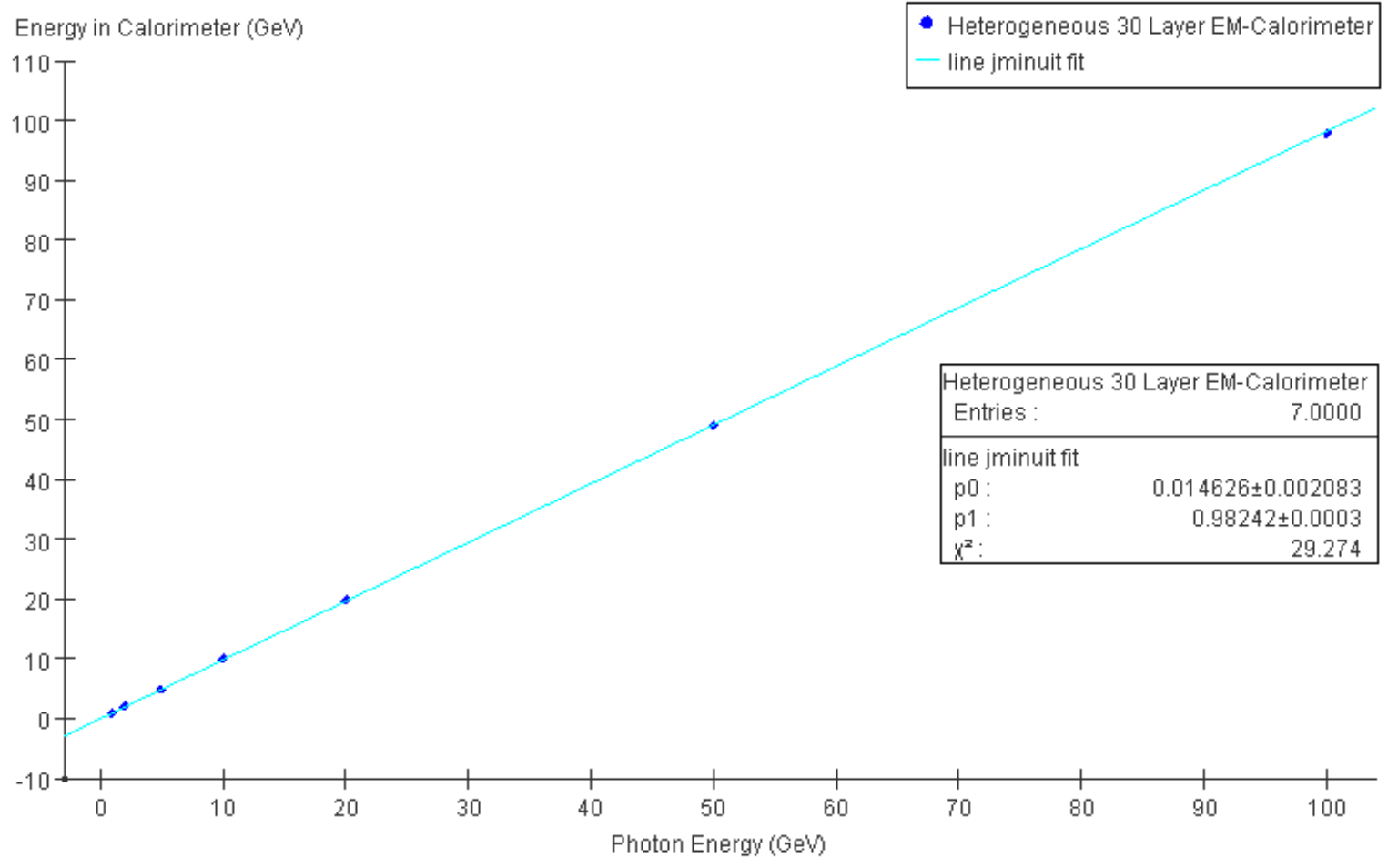

Figure 6. Linearity plot of the total energy in the heterogeneous forty layer EM calorimeter versus the actual energy of a single photon event. The data points are fitted with a linear function of the form $\mathrm{p} 0+\mathrm{p} 1 \mathrm{x}$.

\section{Total Cluster Energy vs. dU or dV}

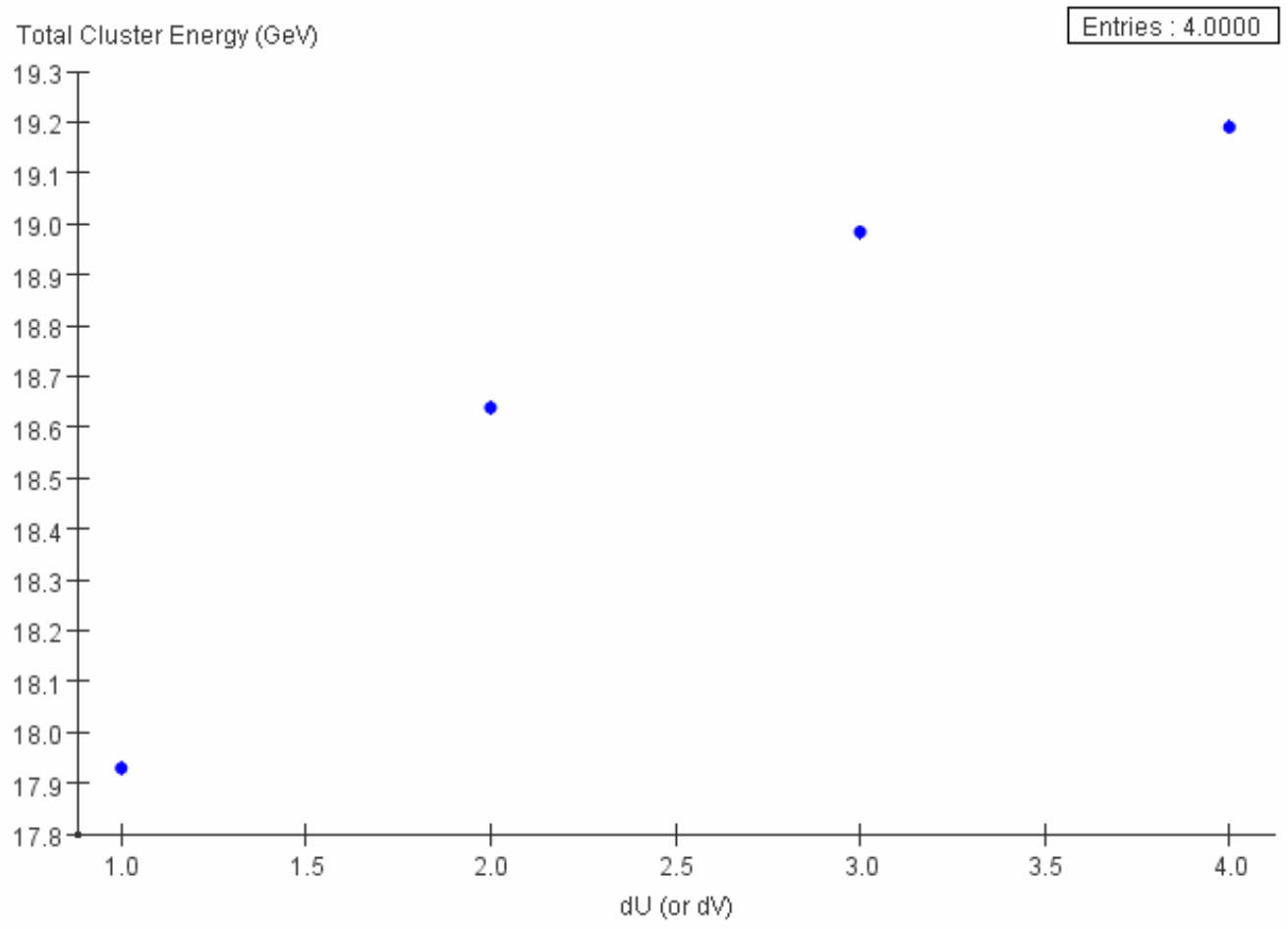

Figure 7. Plot of the total cluster energy for a $20 \mathrm{GeV}$ photon using the nearest neighbor clustering algorithm and varying input parameters $\mathrm{dU}=\mathrm{dV}$. 


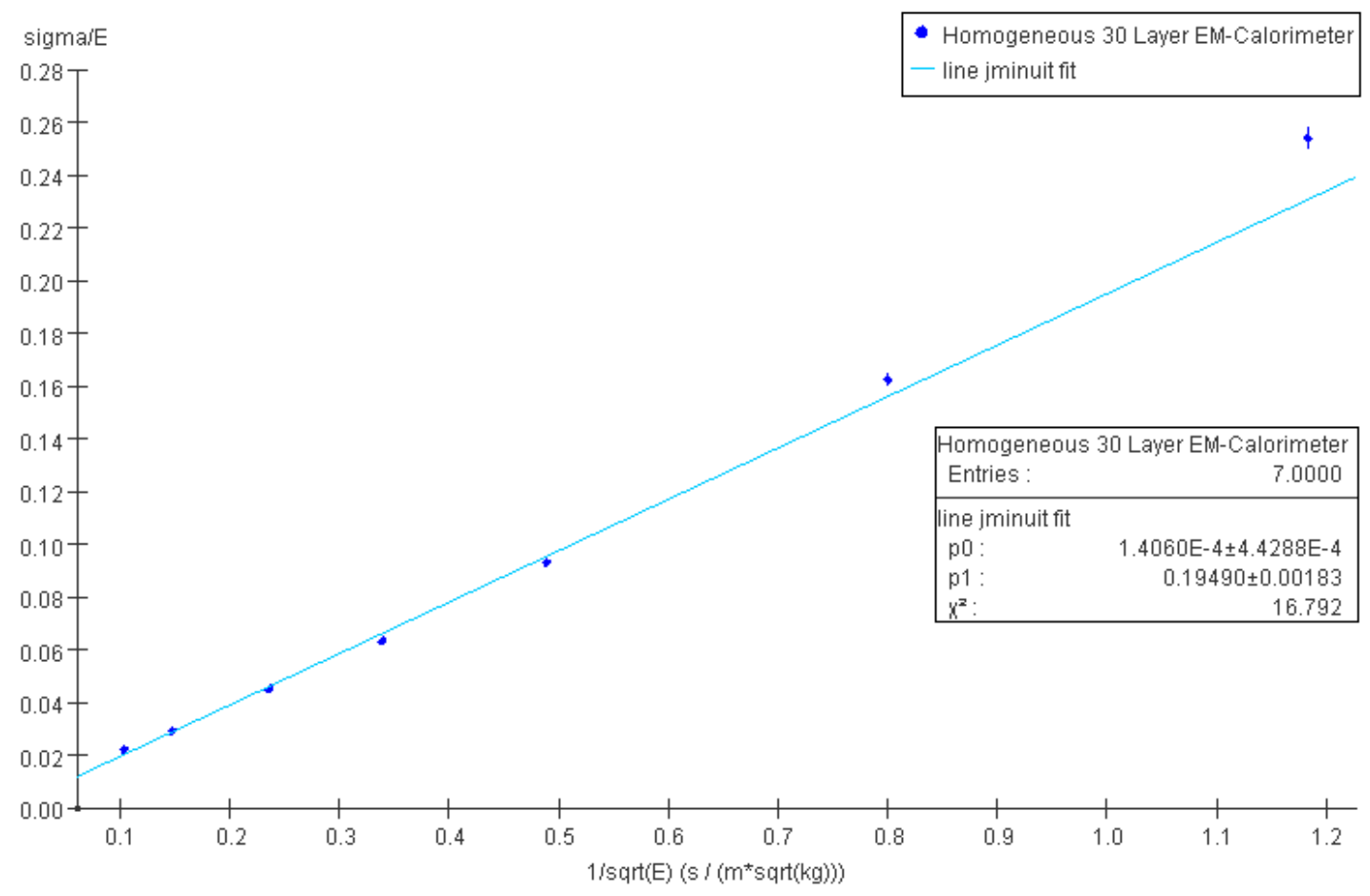

Figure 8. Energy resolution for single photons of varying energy using the homogeneous thirty layer EM calorimeter. The data points are fitted with a linear function of the form $\mathrm{p} 0+\mathrm{p} 1 \mathrm{x}$.

Energy Resolution with Homogeneous $\mathbf{4 0}$ Layer Calorimeter

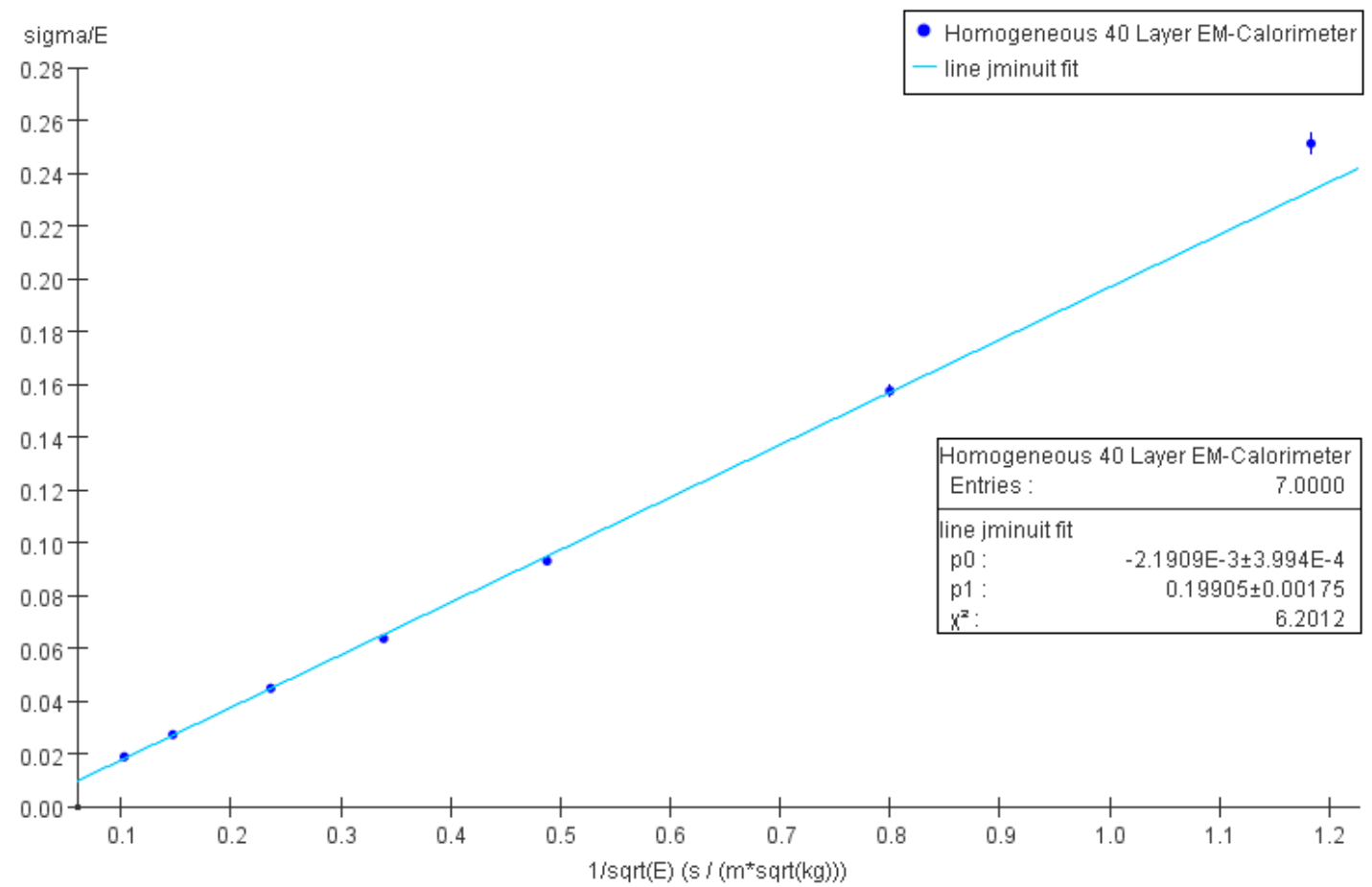

Figure 9. Energy resolution for single photons of varying energy using the homogeneous forty layer EM calorimeter. The data points are fitted with a linear function of the form $\mathrm{p} 0+\mathrm{p} 1 \mathrm{x}$. 
Energy Resolution with Heterogeneous 30 Layer Calorimeter

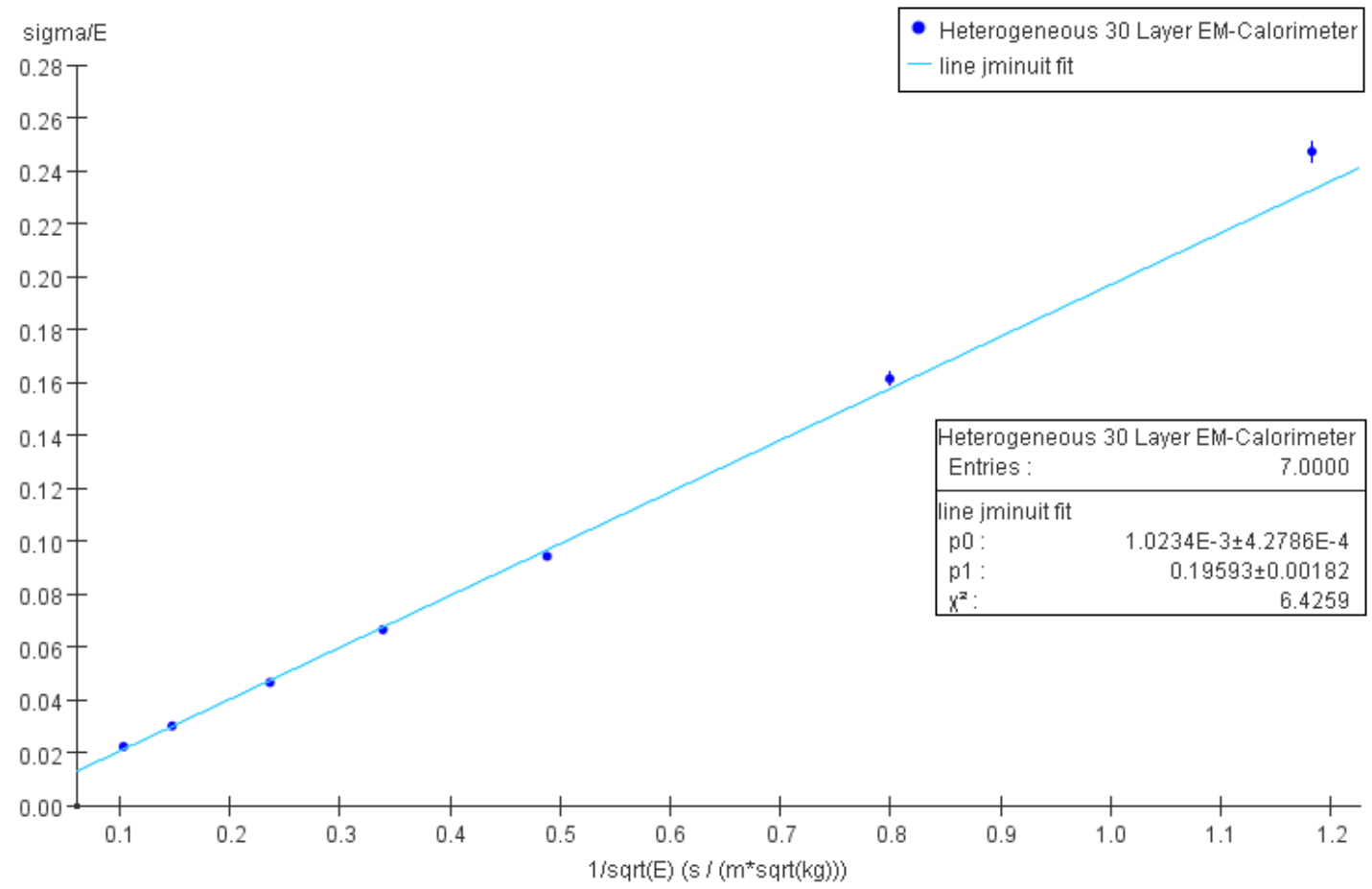

Figure 10. Energy resolution for single photons of varying energy using the heterogeneous thirty layer EM calorimeter. The data points are fitted with a linear function of the form $\mathrm{p} 0+\mathrm{p} 1 \mathrm{x}$. 


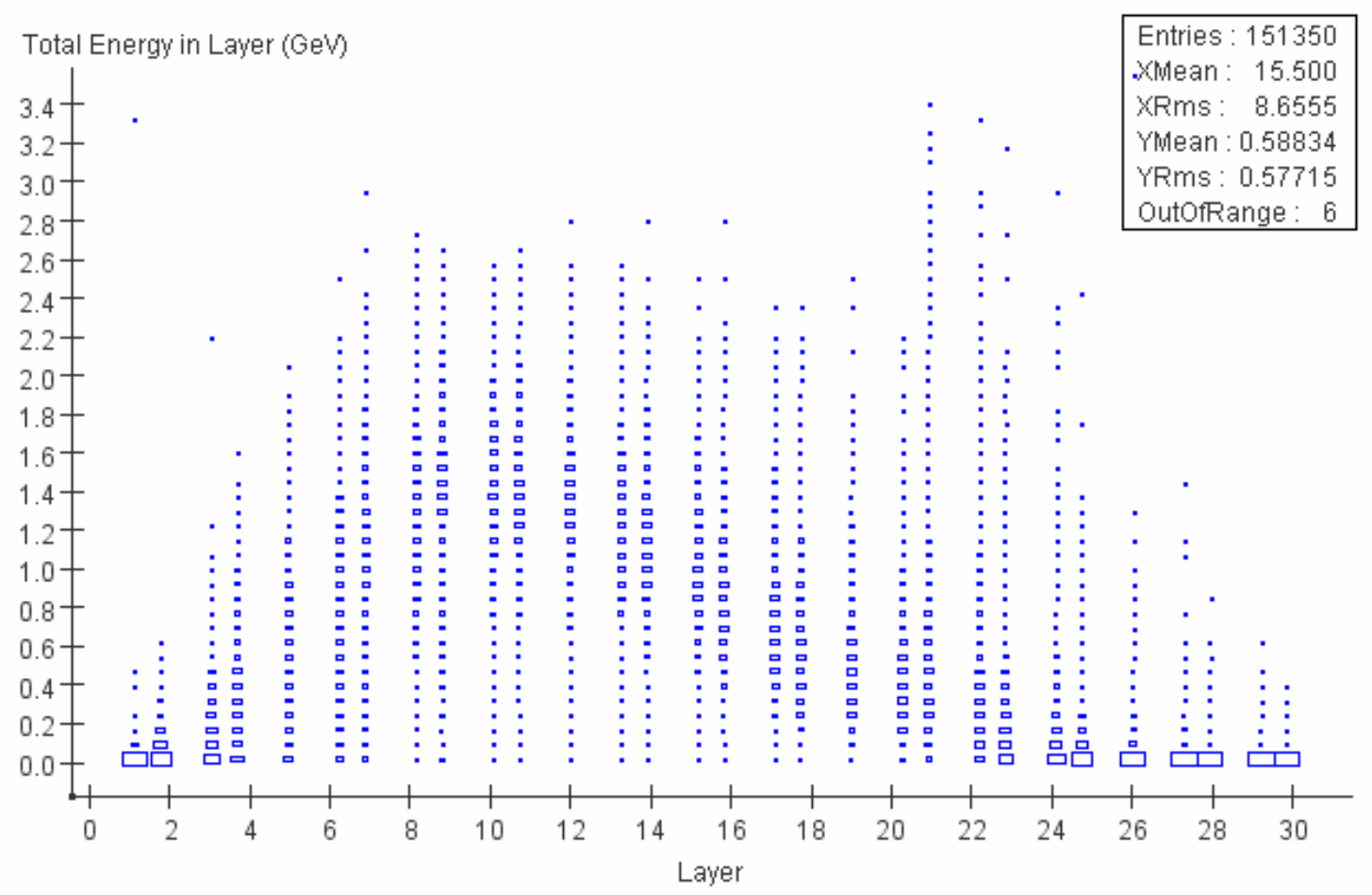

Figure 11. The total energy in each layer of the electromagnetic calorimeter for single $20 \mathrm{GeV}$ photons.

Width vs. Layer

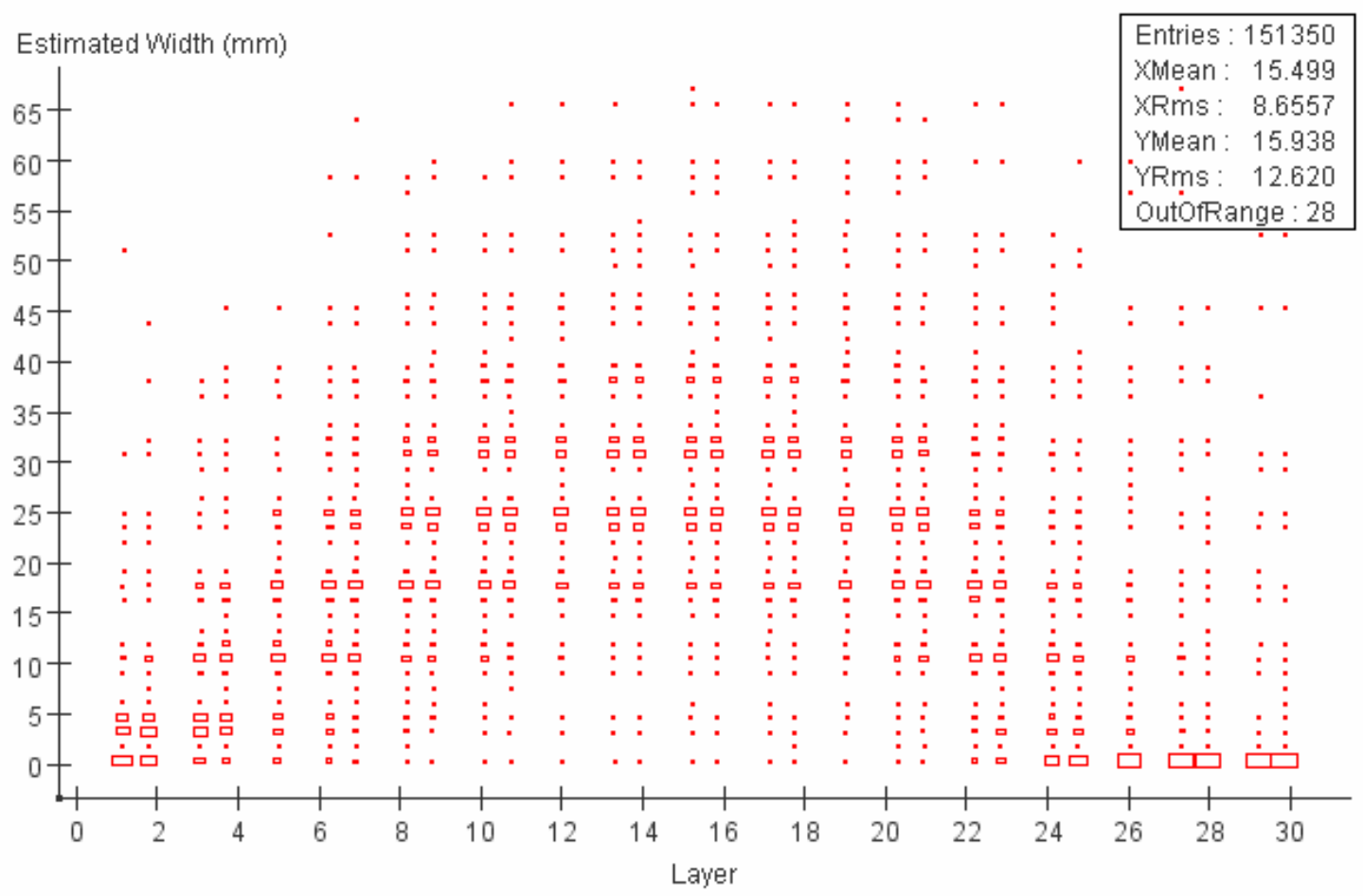

Figure 12. The approximate width of the cluster in each layer of the electromagnetic calorimeter for single $20 \mathrm{GeV}$ photons. 


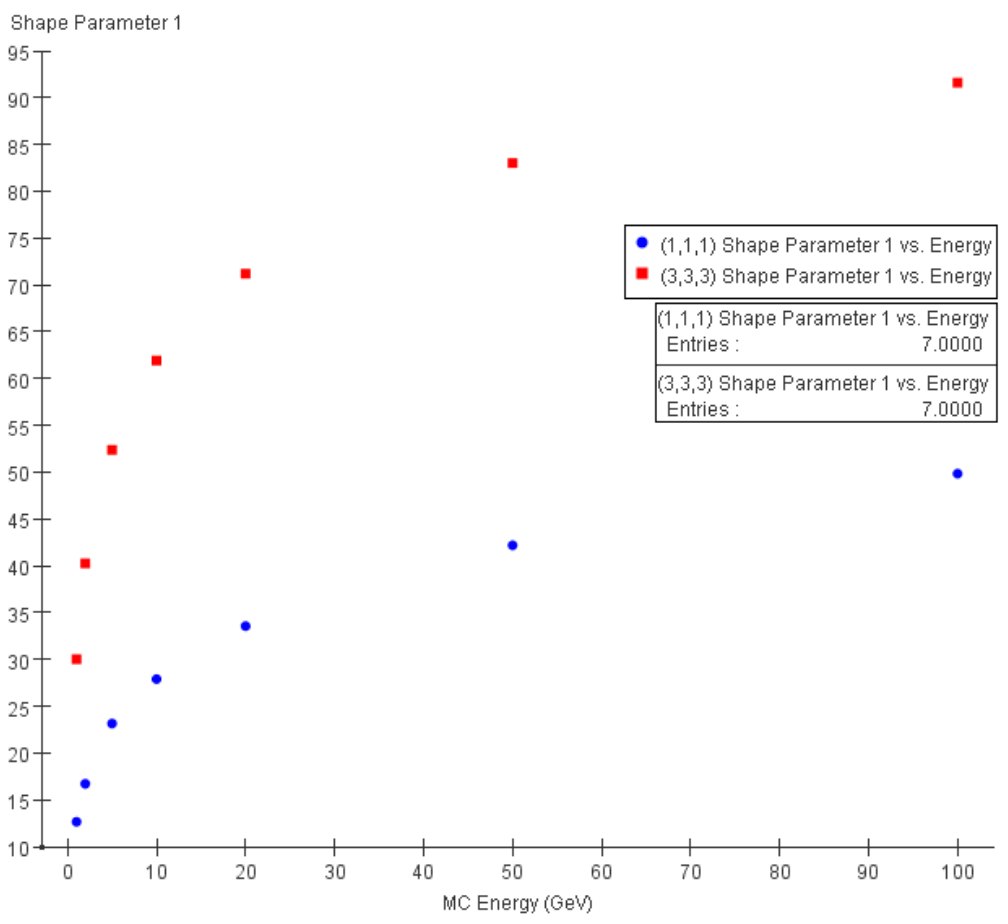

Figure 13. First shape parameter of a single photon cluster versus photon energy with $d U=d V=d l a y e r=1$, labeled $(1,1,1)$ Shape Parameter 1 in the figure, and $d U=d V=$ dlayer $=3$, labeled $(3,3,3)$ Shape Parameter 1 in the figure.

Shape Parameter 2 vs. Energy

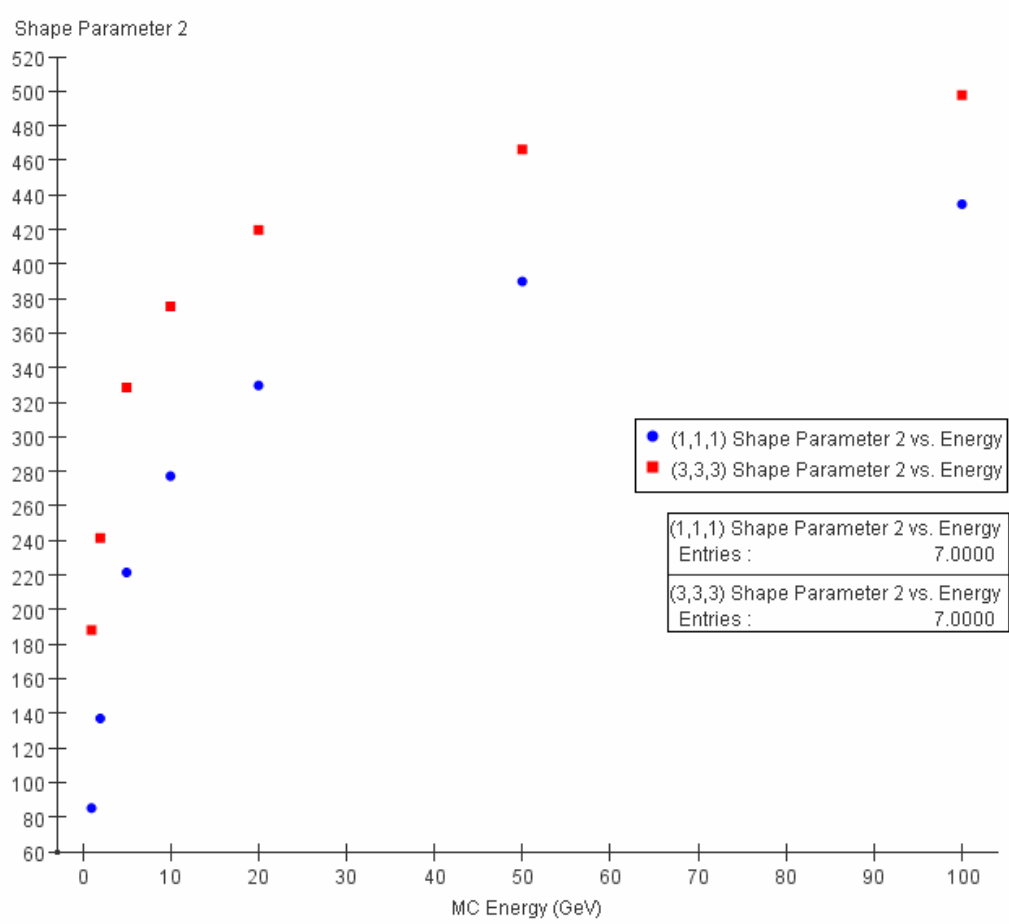

Figure 14. Second shape parameter of a single photon cluster versus photon energy with $d U=d V=d l a y e r=1$, labeled $(1,1,1)$ Shape Parameter 2 in the figure, and $d U=d V=$ dlayer $=3$, labeled $(3,3,3)$ Shape Parameter 2 in the figure. 


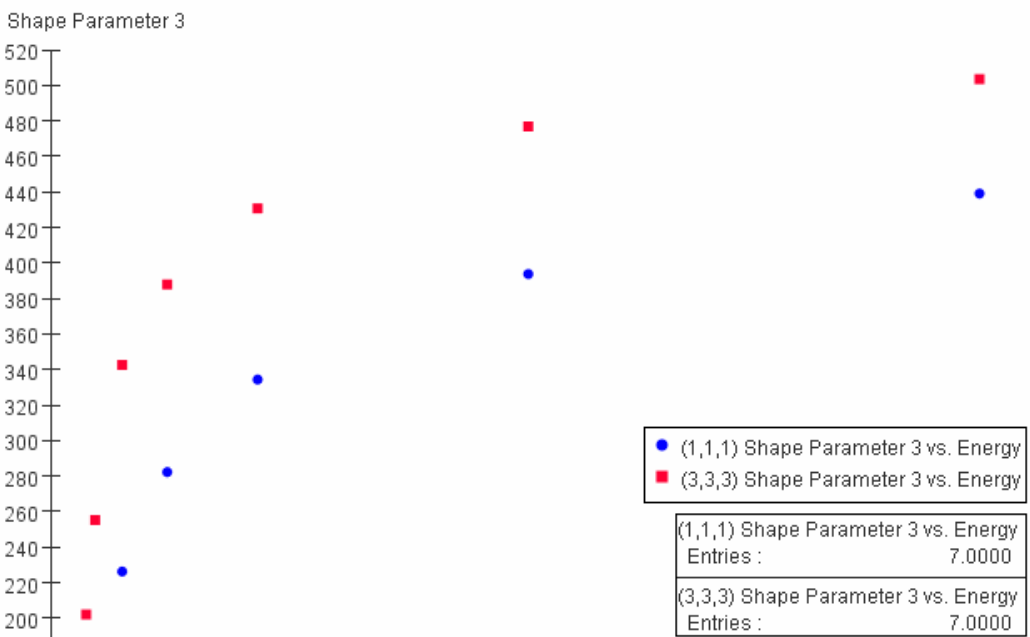

Figure 15. Third shape parameter of a single photon cluster versus photon energy with $d U=d V=$ dlayer $=1$, labeled $(1,1,1)$ Shape Parameter 3 in the figure, and $d U=d V=$ dlayer $=3$, labeled $(3,3,3)$ Shape Parameter 3 in the figure.

Mean Number of Clusters vs. Opening Angle for Two 1GeV Photons

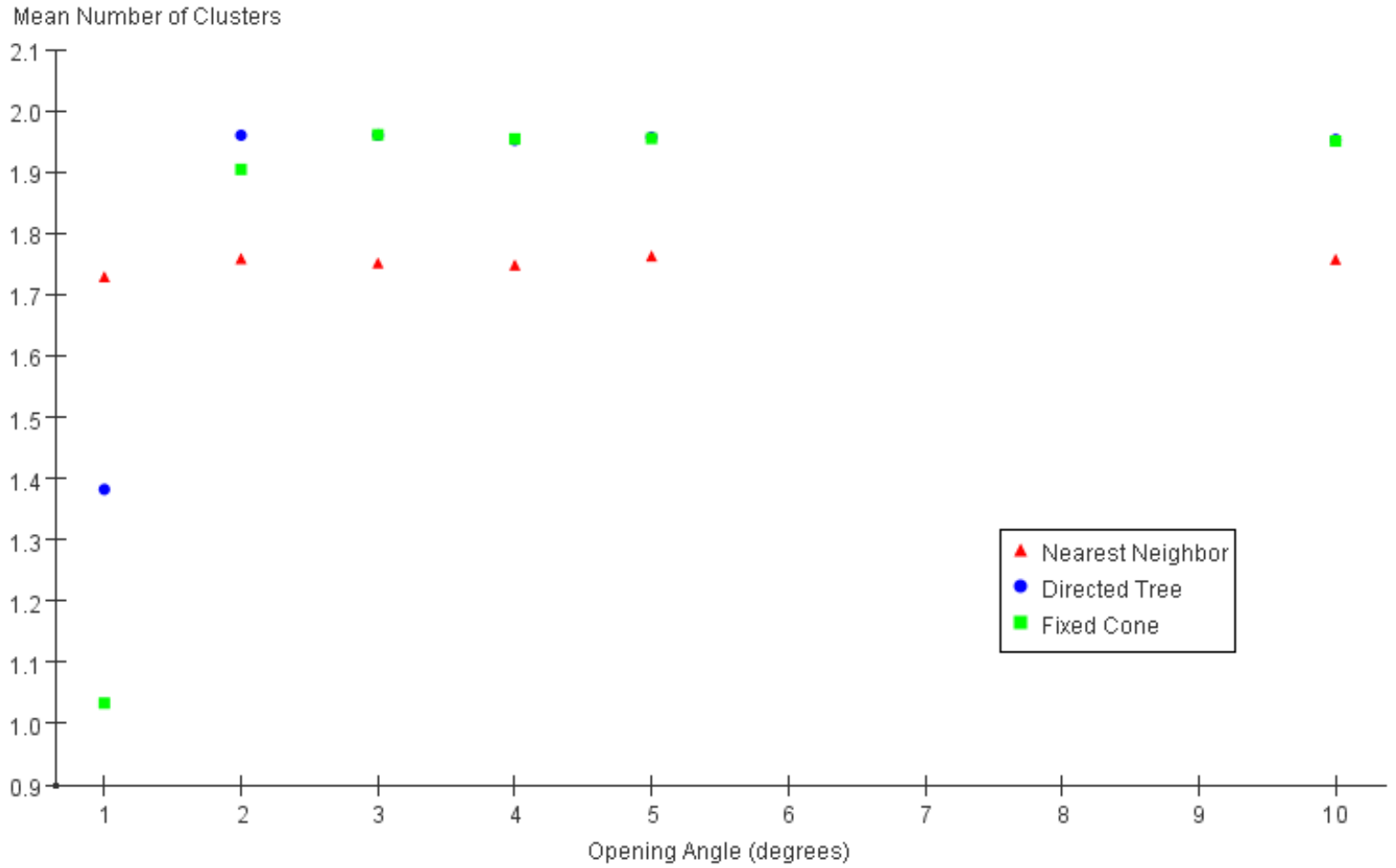

Figure 16. The mean number of clusters detected for two $1 \mathrm{GeV}$ photons with various opening angles using the clustering algorithms: nearest neighbor, directed tree, and fixed cone. 